Bangladesh J. Plant Taxon. 20(1): 95-123, 2013 (June)

(C) 2013 Bangladesh Association of Plant Taxonomists

\title{
TAXONOMY OF THE LEAFY VEGETABLES IN BANGLADESH
}

\author{
Maksuda Khatun, Md. Abul Hassan, Shaikh Nazrul Islam ${ }^{1}$ \\ AND M. OLIUR RAHMAN ${ }^{2}$ \\ Department of Botany, University of Dhaka, Dhaka 1000, Bangladesh
}

Keywords: Leafy vegetables; New reports; Taxonomy; Bangladesh.

\begin{abstract}
Thirty four exploration trips made throughout Bangladesh from 2000 to 2012 resulted in identification of 186 taxa used as leafy vegetables in the country, of which 173 taxa belong to angiosperms and 13 taxa to pteridophytes. Among the angiosperms, Magnoliopsida is represented by 153 taxa under 114 genera and 43 families, whereas Liliopsida is represented by 20 species under 15 genera and 8 families. Pteridophytes are symbolized by 13 species belonging to 10 genera and 10 families. Under each taxon updated nomenclature, vernacular names, habit, representative specimen and area of major consumption of the plant as a leafy vegetable have been provided. Out of 186 leafy vegetables identified in Bangladesh, 140 taxa are wild and 46 are cultivated. Among the cultivated ones 16 species are cultivated only as leafy vegetables and 30 are cultivated for other purposes but also used as leafy vegetables. A total of 61 species have been newly documented as leafy vegetables for Bangladesh.
\end{abstract}

\section{Introduction}

Leafy vegetables are referred to leaves of any plants used as vegetables, sometimes accompanied by tender petioles and shoots. They constitute a major portion of our diet and play an important part in alleviating malnutrition. FAO (2012) has estimated that about 870 million people are chronically undernourished in the period $2010-12$ representing $12.5 \%$ of the global population, or one in eight people. In order to arrest the undernourished situation, much attention has been paid on the exploitation and utilization of unusual plant materials for food (Kawatra et al., 2001; Dini et al., 2005). Leafy vegetables are important protective foods and highly beneficial for the maintenance of health and prevention of diseases as they contain valuable food ingredients. Usually they have no or very little poisonous alkaloids and do not cause any gastrological disturbance when they are consumed as food. The daily intake of at least $100 \mathrm{~g}$ of fresh leafy vegetables is recommended for the adult by nutrition experts (Reddy, 1999). It has been estimated that $100 \mathrm{~g}$ of tropical leafy vegetables can provide $60-140 \mathrm{mg}$ of ascorbic acid, $100 \mathrm{mg}$ of folic acid, 4-7 mg of iron and 200-400 mg of calcium (Saxena, 1999). Traditional leafy vegetables are said to be an invaluable substitute for meat and therefore form important part of daily diets of rural communities in particular.

Over the last decade many studies have shown that fresh leafy vegetables constitute important functional food components by contributing vitamins, iron, folic acid, minerals, biologically active compounds and photosynthetic pigments (Kmiecik et al., 2001; Su et al., 2002; Kimura and Rodriguez-Amaya, 2003). Traditional leafy vegetables have a proven nutritive value in terms of having more protein, minerals, carbohydrate and vitamins than several common vegetables

\footnotetext{
${ }^{1}$ Institute of Nutrition and Food Science, University of Dhaka, Dhaka 1000, Bangladesh.
}

${ }^{2}$ Corresponding author. Email: dr_oliur@yahoo.com 
(Sundriyal and Sundriyal, 2001; Fasuyi, 2006; Orech et al., 2007). Leafy vegetables also contain antioxidants which offer protection against many chronic diseases including heart disease and certain types of cancer (Saxena, 1999).

In Bangladesh, people have a long heritage of taking leafy vegetables. However, very little attempt has been made to study the leafy vegetables of Bangladesh although they constitute a large proportion of the daily diet of the rural dweller of the country (Ali et al., 1977; Sarker and Hossain 2009; Hassan, 2010). Despite the importance of leafy vegetables in the present day human lives, no systematic work has been carried out in Bangladesh to identify and document the plant species. In view of potential beneficial attributes of leafy vegetables, there is a need to explore, identify and document the leafy vegetables of the country. The objectives of the present study are therefore three-fold: i) to make an inventory, and identify the leafy vegetables, ii) to document the leafy vegetables including wild and cultivated ones, and iii) to carry out a detailed systematic study on the leafy vegetables of Bangladesh.

\section{Materials and Methods}

Thirty four field trips were conducted throughout Bangladesh during 2000-2012 to collect fresh plant materials and each field trip consisted of 4-8 days. The areas visited for collection of plant samples include: Bagerhat (Bagerhat Sadar, Mollarhat), Bandarban (Bandarban Sadar, Lama), Barisal (Barisal Sadar), Chittagong (Mirsharai, Sitakundu), Comilla (Comilla Sadar, Daudkandi), Cox's Bazar (Cox’s Bazar Sadar, Teknaf), Dhaka (Nawabganj, Savar), Dinajpur (Birampur, Dinajpur Sadar, Phulbari), Faridpur (Faridpur Sadar, Madhukhali), Gazipur (Gazipur Sadar, Kaliganj, Kapasia, Tongi), Gopalganj (Gopalganj Sadar, Kashiani, Kotalipara, Tungipara), Habiganj (Chunarughat, Madhabpur), Jessore (Jessore Sadar, Jhikargacha, Keshobpur, Manirampur, Sharsha), Jhalokhathi (Jhalokathi Sadar, Rajapur), Jhenaidah (Jhenaidah Sadar, Kaliganj, Kotchandpur, Shailkupa), Khagrachari (Dighinala, Matiranga, Panchari), Khulna (Dighalia, Khulna Sadar, Phultala, Terokhada), Magura (Magura Sadar, Mohammadpur, Shalikha), Manikganj (Saturia, Singair), Moulvi Bazar (Barolekha, Kamalganj, Sreemangal), Munshiganj (Gazaria, Munshiganj Sadar), Mymensingh (Haluaghat, Muktagachha), Narail (Kalia, Lohagara, Narail Sadar), Narayanganj (Fatullah, Siddhirganj), Natore (Natore Sadar, Singra), Netrakona (Durgapur, Khaliajuri), Patuakhali (Kalapara, Patuakhali Sadar), Rajbari (Baliakandi, Pangsha, Rajbari Sadar), Rajshahi (Bagha, Bagmara, Godagari, Puthia), Rangamati (Kaptai, Rangamati Sadar), Sherpur (Jhenaigathi, Sherpur Sadar), Sunamganj (Jagannathpur, Sunamganj Sadar), Sylhet (Sylhet Sadar) and Tangail (Madhupur). Collection of fresh materials was made from local markets, village areas and forest lands. Prior to collection, assistance from local informants was taken regarding the use of plants as leafy vegetables. Local vegetable markets were also surveyed to record marketable items. The collected samples were processed following the standard herbarium technique (Hyland, 1972). Some samples were also kept in liquid preservative.

The collected specimens were critically studied and identified in the Dhaka University Salar Khan Herbarium (DUSH) by matching with the identified specimens housed at DUSH and DACB (Bangladesh National Herbarium), and with the help of standard literatures (Hooker, 1872-1897; Prain, 1903; Khan, 1972-1987; Dassanayake and Fosberg, 1980-1985; Khan and Halim, 1987; Khan and Rahman, 1989-2002). Nomenclatures have been updated using Siddiqui et al. (20072008), Ahmed et al. (2008-2009), and Rashid and Rahman (2011, 2012). The angiosperm families followed that of Cronquist (1981), while pteridophyte families are arranged in an alphabetical order. Under each family the genera and species have been arranged alphabetically. Updated nomenclature, vernacular names (Eng. = English, Beng. = Bangla), habit, representative specimen (only one cited because of page constraint) and area of major consumption have been furnished 
under each taxon. All voucher specimens have been deposited at DUSH in the Department of Botany, University of Dhaka, Bangladesh.

\section{Results and Discussion}

A total of 186 leafy vegetable taxa have been identified in Bangladesh of which 173 belong to angiosperms, and 13 to pteridophytes. Among the angiospermic taxa Magnoliopsida is represented by 153 and Liliopsida is represented by 20 taxa (Table 1 ).

Table 1. Number of taxa of leafy vegetables recorded in Bangladesh.

\begin{tabular}{lcccc}
\hline Taxa & Magnoliopsida & Liliopsida & Pteridophyta & Total \\
\hline Families & 43 & 8 & 10 & 61 \\
Genera & 114 & 15 & 10 & 139 \\
Species & 153 & 20 & 13 & 186 \\
\hline
\end{tabular}

The taxonomic enumeration of the leafy vegetables is briefly described below.

\section{MAGNOLIOPSIDA}

\section{Piperaceae C. A. Agardh (1825)}

1. Peperomia pellucida (L.) H. B. K., Nov. Gen. Sp. 1: 64 (1815). Vernacular names: Pepper Elder (Eng.), Luchi Pata (Beng.), Samol-hapang (Garo).

A fleshy annual herb. Representative specimen: Netrakona: Boheratoli, 8.10.2001, M. Khatun 10. Area of major consumption: Netrakona district.

2. Piper longum L., Sp. Pl.: 29 (1753). Vernacular names: Long Pepper (Eng.), Pipla-mul, Pipul, Pipul Morich (Beng.).

A perennial herb. Representative specimen: Gopalganj: Tatulia, 3.3.2007, M. Khatun 428. Area of major consumption: Rajbari district.

\section{Moraceae Link (1831)}

3. Ficus benghalensis L., Sp. Pl.: 1059 (1753). Vernacular names: Banyan Tree (Eng.), Bot, Botgachh (Beng.), Jalong (Khasia).

A large tree. Representative specimen: Moulvi Bazar: Madhabpunji, Madhabkundu, 5.5.2003, M. Khatun 274. Area of major consumption: Moulvi Bazar district.

4. F. carica L., Sp. Pl. 2: 1059 (1753). Vernacular names: Common Fig, European Fig (Eng.), Anjir, Dumur (Beng.), Soluya (Khasia).

A large shrub or small tree. Representative specimen: Moulvi Bazar: Madhabkundu, 3.5.2003, M. Khatun 276. Area of major consumption: Moulvi Bazar district.

\section{Nyctaginaceae A. L. de Jussieu (1789)}

5. Boerhavia diffusa L., Sp. Pl.: 3 (1753). Vernacular names: Pigweed, Spreading Hog-weed (Eng.), Punarnava (Beng.)

A perennial, creeping or climbing herb. Representative specimen: Rajbari: Olangapur, 8.2.2004, M. Khatun 421. Area of major consumption: Rajbari district.

\section{Aizoaceae Rudolphi (1830)}

6. Sesuvium portulacastrum (L.) L., Syst. ed. 10: 1058 (1759). Vernacular names: Shoreline Sea Purslane (Eng.), Nuna Shak (Beng.), Phru-bawn (Rakhain). 
A perennial herb. Representative specimen: No specimen was collected, but information was gathered from the local people. Area of major consumption: Patuakhali district.

\section{Chenopodiaceae Ventenant (1799)}

7. Beta vulgaris L., Sp. Pl. 1: 222 (1753). Vernacular names: Garden Beet, Common Beet (Eng.), Beet, Palak (Beng.).

A biennial herb. Representative specimen: Jhalokathi: Rajapur, 11.4.2010, M. Khatun 588. Area of major consumption: Dhaka district.

8. Chenopodium album L., Sp. Pl. 1: 219 (1753). Vernacular names: Pigweed (Eng.), Batua Shak (Beng.).

An annual herb. Representative specimen: Rajbari: Salmara, 8.2.2004, M. Khatun 402. Area of major consumption: Rajbari district.

9. Spinacia oleracea L., Sp. Pl. 1: 219 (1753). Vernacular names: White Goosefoot (Eng.), Palong Shak (Beng.), Mui-yaa-bawn (Rakhain).

A small, annual herb. Representative specimen: Rajshahi: Binodpur, 13.9.2001, M. Khatun 51. Area of major consumption: Dhaka district.

6. Amaranthaceae A. L. de Jussieu (1789)

10. Achyranthes aspera L., Sp. Pl. 1: 204 (1753).Vernacular names: Prickly Chaff-flower (Eng.), Apang, Upatlengra (Beng.), Longra (Santal).

A perennial, erect herb. Representative specimen: Dinajpur: Mukundupur, 16.6.2001, M. Khatun 76. Area of major consumption: Dinajpur, Mymensingh and Comilla districts.

11. Aerva sanguinolenta (L.) Bl., Bijdr.: 547 (1826). Vernacular names: Nuriya, Lal Apang (Beng.), Nenga (Chakma).

A perennial herb. Representative specimen: Moulvi Bazar: Adampur bit, 3.5.2003, M. Khatun 194. Area of major consumption: Sunamganj and Moulvi Bazar districts.

12. Alternanthera bettzickiana (Regel) Voss Nichols., III. Dict. Grad. 1: 59 (1884). Vernacular names: Joyweed (Eng.), Nun-khuta Shak (Beng.).

A perennial herb. Representative specimen: Patuakhali: Kolapara, 23.5.2008, M. Khatun 475. Area of major consumption: Patuakhali district.

13. A. paronichyoides St. Hil., Voy. Distr. Diamans Bresil. 2: 439 (1833). Vernacular names: Smooth Chaff-flower (Eng.), Jhuli Khata (Rakhain).

A perennial mat-forming herb. Representative specimen: Patuakhali: Kalachandpara, 23.5.2008, M. Khatun 476. Area of major consumption: Patuakhali district.

14. A. philoxeroides (Mart.) Griseb., Symb. Argent. in Abh. Ges. Wiss. Gott. 24: 36 (1879). Vernacular names: Alligator Weed (Eng.), Malancha Shak (Beng.), Shergiti (Santal).

A perennial, polymorphic herb. Representative specimen: Dinajpur: Noiyabad, 16.9.2001, M. Khatun 41. Area of major consumption: Dinajpur district.

15. A. sessilis (L.) R. Br. ex Roem. \& Schult., Syst. 5: 554 (1819). Vernacular names: Sessile Joyweed (Eng.), Chanchi, Sachishak (Beng.), Garundi (Garo).

Annual or perennial herb. Representative specimen: Rajshahi: Binodpur, 13.9.2001, M. Khatun 37. Area of major consumption: Rajshahi district. 
16. Amaranthus blitum L., Sp. Pl. ed. 1: 990 (1753). Vernacular names: Purple Amaranth (Eng.), Natiyasag (Beng.).

A tall, glabrous, succulent herb. Representative specimen: Narail: Lohagara, 15.9.2007, M. Khatun 458. Area of major consumption: Narail district.

17. A. spinosus L., Sp. Pl. ed. 1: 991 (1753). Vernacular names: Spiny Amaranth (Eng.), Kantanotey, Kantadenga (Beng.), Katakailpha, Kuriakanta (Tripura).

An annual herb. Representative specimen: Dhaka: Uttara, 14.1.2002, M. Khatun 168. Area of major consumption: Dhaka district.

18. A. tricolor L., Sp. Pl. ed. 1: 989 (1753). Vernacular names: Joseph’s Coat (Eng.), Lal Shak, Dengua (Beng.), Puspoo (Santal).

An annual herb. Representative specimen: Jessore: Barobazar, 5.1.2004, M. Khatun 383. Area of major consumption: Mostly in urban areas.

19. A. viridis L., Sp. Pl. ed. 2: 1405 (1763). Vernacular names: Green Amaranth, (Eng.), Notey, Notey Shak (Beng.).

An annual herb. Representative specimen: Rajbari: Konagram, 8.2.2004, M. Khatun 416. Area of major consumption: Rajbari district.

20. Celosia argentea L., Sp. Pl. 1: 205 (1753). Vernacular names: Cock’s Comb (Eng.), Sada Moragphul (Beng.), Thinthinga (Garo), Thanthania (Rakhain).

An erect, annual herb. Representative specimen: Netrakona: Bijoypur, 8.1.2000, M. Khatun 9. Area of major consumption: Patuakhali and Netrakona districts.

21. C. cristata L., Sp. Pl. 1: 235 (1753). Vernacular names: Crested Cock’s Comb (Eng.), Morogful (Beng.), Shibjota (Garo).

A much branched herb. Representative specimen: Patuakhali: Kalachanpara, 23.5.2008, M. Khatun 480. Area of major consumption: Netrakona and Patuakhali districts.

22. Digera muricata (L.) Mart. in Nov., Acad. Caes. Leop. Carol. 13(1): 285 (1826). Vernacular names: False Amaranth (Eng.), Boutibon Shak (Beng.), Latamouri (Garo).

An annual herb. Representative specimen: Khagrachari: Golabari, 7.7.2003 M. Khatun 328. Area of major consumption: Netrakona and Khagrachari districts.

23. Psilotrichum ferrugineum (Roxb.) Moq. in DC., Prod. 13(2): 279 (1849). Vernacular names: Rokto-sirinch (Beng.), Puti Shak (Santal).

An annual herb. Representative specimen: Dinajpur: Noabad, 16.9.2001, M. Khatun 78. Area of major consumption: Dinajpur district.

7. Portulacaceae A. L. de Jussieu (1789)

24. Portulaca oleracea L., Sp. Pl.: 445 (1753). Vernacular names: Purslane (Eng.), Bara Lunia, Kulfi, Lunia Shak (Beng.), Tee-jey-shey (Marma).

An annual herb. Area of major consumption: Dhaka district. Representative specimen: Dhaka: Shambazar, 14.1.2002, M. Khatun 142.

25. P. quadrifida L., Mant. Pl. 1: 73 (1767). Vernacular names: Pot Purslane, Small-leaved Purslane (Eng.), Chhota Lunia, Munia Shak (Beng.).

A small, prostrate, annual herb. Representative specimen: Rajshahi: Shaheb Bazar, 13.9.2001, M. Khatun 54. Area of major consumption: Dhaka and Rajshahi districts. 
8. Basellaceae Moquin-Tandon (1840)

26. Basella alba L., Sp. Pl. 1: 272 (1753). Vernacular names: Indian Spinach (Eng.), Puishak (Beng.).

A much branched, fleshy herb. Representative specimen: Dhaka: Diabari, 14.1.2002, M. Khatun 131. Area of major consumption: Dhaka district.

9. Molluginaceae Hutchinson (1926)

27. Glinus oppositifolius (L.) A. DC., Bull. Herb. Boiss. 2(1): 522 (1901). Vernacular names: Gimashak (Beng.), Dima Tita (Koach, Santal).

A diffusely branched, annual herb. Representative specimen: Dinajpur: Mukundopur, 16.9.2001, M. Khatun 85. Area of major comsumption: Dinajpur and Sherpur districts.

28. Mollugo pentaphylla L., Sp. Pl. 1: 89 (1753). Vernacular names: Mollugo (Eng.), Khetpapra (Beng.), Tita Shak (Garo).

An annual herb. Representative specimen: Tangail: Madhupur, Pirgacha, 18.4.2002, M. Khatun 176. Area of major comsumption: Tangail district.

10. Caryophyllaceae A. L. de Jussieu (1789)

29. Polycarpon prostratum (Forssk.) Aschers. \& Schweinf., Oesterr. Bot. Zeitscher 39: 128 (1889). Vernacular names: Ghimashak (Beng.), Beng-bong-jathong (Koach).

A dichotomously branched herb. Representative specimen: Sherpur: Gajni, 31.10.2009, M. Khatun 520. Area of major consumption: Sherpur district.

30. Stellaria wallichiana Benth. ex Haines, Bull. Misc. Inf. Kew 1920: 66 (1920). Vernacular names: Sada Fulki, Tara (Beng.), Murmuri Shak (Koach).

A herb. Representative specimen: Moulvi Bazar: Barolekha, Madhabkundu, 4.5.2003, M. Khatun 272. Area of major consumption: Moulvi Bazar district.

11. Polygonaceae A. L. de Jussieu (1789)

31. Ampelygonum chinense (L.) Lindley, Bot. Reg. 24: 63 (1838). Vernacular names: Trailing Smartweed (Eng.), Mohicharan Shak (Beng.), Mono-eja-dar (Chakma).

A perennial herb. Representative specimen: Moulvi Bazar: Madhabpunji, 4.5.2003, M. Khatun 277. Area of major consumption: Moulvi Bazar district.

32. A. microcephalum (D. Don) Hassan, Bangladesh J. Bot. 22(1): 4 (1993). Vernacular names: Hilly Smartweed (Eng.), Madhusilum Shak (Beng.), Ambimikchip (Garo).

A perennial herb. Representative specimen: Netrakona: Bijoypur, 8.10.2001, M. Khatun 06. Area of major consumption: Netrakona district.

33. A. salarkhanii Hassan, Bangladesh J. Bot. 20(2): 245 (1991). Vernacular names: Hilly Smartweed (Eng.), Giri Shobhan Shak (Beng.), Lambak (Khasia).

An undershrub. Representative specimen: Rangamati: Kaptai, Shilchari, 6.7.2003, M. Khatun 350. Area of major consumption: Rangamati district.

34. Persicaria tomentosa (Willd.) Sasaki, List Pl. Form.: 170 (1928). Vernacular names: Hairy Knotweed (Eng.), Pani-bishkatali (Beng.), Hagra (Mandi).

A perennial, aquatic herb. Representative specimen: Tangail: Madhupur, Pirgacha, 18.4.2002, M. Khatun 177. Area of major consumption: Tangail district. 
35. Polygonum effusum Meissn. in DC., Prodr. 14: 93 (1857). Vernacular names: Knotweed (Eng.), Raniphul, Chemtisag (Beng.), Kuttasunga Gas (Koach).

An annual herb. Representative specimen: Gazipur: Gozaripara, 4.12.2009, M. Khatun 552. Area of major consumption: Gazipur district.

36. P. plebeium R. Br., Prodr.: 420 (1810). Vernacular names: Small Knotweed (Eng.), Khudibishkatali (Beng.), Khumchak, Gang-sum (Khasia).

A prostrate annual herb. Representative specimen: Sylhet: Madhabkundu, 4.5.2003; M. Khatun 279. Area of major consumption: Habiganj district.

37. Rumex dentatus L., Mant. Pl. 2: 226 (1771). Vernacular names: Toothed Dock (Eng.), Bon Tak-palong (Beng.).

An annual herb. Representative specimen: Rajshahi: Shaheb Bazar, 13.9.2001, M. Khatun 47. Area of major consumption: Rajshahi district.

38. R. maritimus L., Sp. Pl. 1: 335 (1753). Vernacular names: Golden Dock (Eng.), Bon Palong (Beng.).

An annual herb. Representative specimen: Natore: Singair, 14.9.2001, M. Khatun 61. Area of major consumption: Dhaka and Natore districts.

39. R. vesicarius L., Sp. Pl. 1: 336 (1753). Vernacular names: Rosy Dock (Eng.), Tok Palong, Chuka Palong (Beng.).

An annual, glabrous herb. Representative specimen: Rajshahi: Meharchandi, 13.9.2001, M. Khatun 53. Area of major consumption: Rajshahi and Sylhet districts.

12. Elaeocarpaceae A. P. de Candolle (1824)

40. Elaeocarpus floribundus Blume, Bijdr.: 120 (1825). Vernacular names: Indian Olive (Eng.), Jalpai (Beng.), Uthethamo (Marma).

A medium-sized to large tree. Representative specimen: Bandarban: Bandarban Sadar, 6.3.2012, M. Khatun 618. Area of major consumption: Bandarban district.

13. Tiliaceae A. L. de Jussieu (1789)

41. Corchorus capsularis L., Sp. Pl. : 529 (1753). Vernacular names: Jute (Eng.), Deshi Pat, Bagi Pat (Beng.).

An erect, annual herb. Representative specimen: Faridpur: Mokshedpur, 9.7.2008, M. Khatun 412. Area of major consumption: Faridpur district.

42. C. olitorius L., Sp. Pl. : 529 (1753). Vernacular names: Tossa Jute (Eng.), Tosha Pat, Lalita Pat, Deo Pat (Beng.).

An annual, erect herb. Representative specimen: Dhaka: Bosila, 15.10.2002, M. Khatun 238. Area of major consumption: Dhaka district.

14. Sterculiaceae Bartling (1830)

43. Sterculia villosa Roxb. ex Smith in Rees, Cyc. 34: No. 16 (1816). Vernacular names: Udal (Beng.), Lambuk (Tripura).

A large, deciduous tree. Representative specimen: Moulvi Bazar: Madhabpungi, Madhabkundu, 5.5.2003, M. Khatun 280. Area of major consumption: Bandarban and Moulvi Bazar districts. 
15. Malvaceae A. L. de Jussieu (1789)

44. Hibiscus cannabinus L., Syst. Nat. ed. 10, 2: 1149 (1759). Vernacular names: Kenaf Hemp, Decan Hemp (Eng.), Mesta Pat (Beng.), Dare Kudrum (Garo).

A herb with prickly stem. Representative specimen: Patuakhali: Kuakata, 23.5.2008, M. Khatun 485. Area of major consumption: Patuakhali district.

45. H. sabdariffa L., Sp. Pl.: 695 (1753). Vernacular names: Kenaf Hemp (Eng.), Mesta Pat (Beng.), Chukair (Garo), Arak (Rakhain).

An erect, annual herb. Representative specimen: Tangail: Madhupur, 18.4.2002, M. Khatun 175. Area of major consumption: Tangail district.

46. H. surattensis L., Sp. Pl.: 696 (1753). Vernacular names: Wild Sour (Eng.), Mikhri (Garo), Maik Shak (Khasia).

An annual herb. Representative specimen: Tangail: Madhupur, Chonia, 18.4.2002, M. Khatun 175. Area of major consumption: Tangail district.

47. Malva verticillata L., Sp. Pl.: 689 (1753). var. rafiqii Abedin in Nasir \& Ali (Eds), Fl. W. Pak. 130: 43 (1979). Vernacular names: Whorled Malva (Eng.), Napha, Napa (Beng.).

An annual herb. Representative specimen: Dinajpur: Dinajpur proper, 16.9.2001, M. Khatun 83. Area of major consumption: Dinajpur and Rangpur districts.

16. Cucurbitaceae A. L. de Jussieu (1789)

48. Benincasa hispida (Thunb.) Cogn. in DC., Monogr. Phan. 3: 513 (1881). Vernacular names: Wax Gourd (Eng.), Chalkumra (Beng.).

An annual, hispid, climbing herb. Representative specimen: Jessore: Birampur, 5.1.2004, M. Khatun 391. Area of major consumption: Dhaka and Jessore districts.

49. Coccinia grandis (L.) Voigt, Hort. Suburb. Calc.: 59 (1854). Vernacular names: Ivy Gourd (Eng.), Telakucha (Beng.).

A perennial, climbing herb. Representative specimen: Natore: Shingra, 14.4.2001, M. Khatun 57. Area of major consumption: Gazipur district.

50. Cucurbita maxima Duch. ex Lamk., Encycl. 2: 151 (1786). Vernacular names: Pumpkin (Eng.), Mistikumra, Mithakumra (Beng.).

An annual, climbing herb. Representative specimen: Rajshahi: Binodpur, 13.9.2001, M. Khatun 41. Area of major consumption: Rajshahi district.

51. Lagenaria siceraria (Molina) Standl., Publ. Field Mus. Nat. Hist. Chicago, B. Ser. 3: 435 (1930). Vernacular names: Bottle Gourd (Eng.), Lau (Beng.), Boo-sthie (Rakhain).

A large, annual, climbing herb. Representative specimen: Dhaka: Uttarbadda, 15.10.2002, M. Khatun 235. Area of major consumption: Dhaka district.

52. Luffa cylindrica (L.) M. Roem., Synops. 2: 63 (1846). Vernacular names: Sponge Gourd (Eng.), Dhundul (Beng.), Mree-u-sthie (Rakhain).

An extensive climbing herb. Representative specimen: Jessore: Khoertola, 5.1.2004, M. Khatun 392. Area of major consumption: Jessore district.

53. Momordica charantia L. var. muricata (Willd.) Chakravarty, Fasc. Fl. Ind. 2: 92 (1982). Vernacular names: Bitter Melon (Eng.), Uchchhey (Beng.), Gang-khera-apang (Rakhain). 
An annual, climbing herb. Representative specimen: Gopalganj: Vatiapara, 3.3.2007, M. Khatun 426. Area of major consumption: Gopalganj district.

54. Trichosanthes anguina L., Sp. Pl. 1: 1008 (1753). Vernacular names: Snake Gourd (Eng.), Chichinga (Beng.), Mring-bawn (Rakhain).

An annual, climbing herb. Representative specimen: Noakhali: Begumganj, 7.5.2003, M. Khatun 241. Area of major consumption: Noakhali and Jessore districts.

55. T. dioica Roxb., Fl. Ind. 3: 701 (1832). Vernacular names: Pointed Gourd (Eng.), Patal (Beng.), Pee-tho-sthie (Rakhain).

An annual, climbing herb. Representative specimen: Rajshahi: Binodpur, 13.9.2001, M. Khatun 44. Area of major consumption: Rajshahi district.

56. Zehneria japonica (Thunb.) H. Y. Liu., Bull. Nat. Mus. Nat. (Taiwan) 1: 40 (1989). Vernacular name: White-fruited Creeping Cucumber (Eng.), Herana Shak (Chakma, Khasia).

An annual, climbing herb. Representative specimen: Rangamati: Kaptai, Digholchari, 7.7.2003, M. Khatun 346. Area of major consumption: Rangamati district.

57. Z. scabra (L. f.) Sond. in Harv. \& Sond., Fl. Cap. 2: 486 (1862). Vernacular names: South African Zehneria (Eng.), Rakhal Sasa (Beng.), Kolakachu (Koch).

An annual, climbing herb. Representative specimen: Sherpur: Halchati, 1.11.2009, M. Khatun 527. Area of major consumption: Sherpur district.

\section{Begoniaceae C. A. Agardh (1825)}

58. Begonia barbata Wall. ex. A. DC., Prodr. 15(1) : 348 (1864). Vernacular name: Tokpata (Tripura).

A herb with creeping rootstock. Representative specimen: Moulvi Bazar: Sreemangal, 6.5.2003, M. Khatun 304. Area of major consumption: Moulvi Bazar district.

18. Brassicaceae Burnett (1835)

59. Brassica juncea (L.) Czerniak., Consp. Fl. Chark. : 8 (1859). Vernacular names: Indian Mustard, Brown Mustard (Eng.), Raisarisha, Jhuni, Chanchi (Beng.).

An annual herb. Representative specimen: Manikganj: Singair, 13.9.2002, M. Khatun 184. Area of major consumption: Dhaka and Sylhet districts.

60. B. napus L., Sp. Pl. 2: 666 (1753). Vernacular names: Colza, Rape (Eng.), Magi, Togi, Sarisha (Beng.).

An annual herb. Representative specimen: Rajshahi: Meharchandi, 13.9.2001, M. Khatun 41. Area of major consumption: Dhaka district.

61. B. oleracea var. capitata L., Sp. Pl. 2: 667 (1753). Vernacular names: Cabbage, Headed Cabbage (Eng.), Bandhakapi (Beng.).

An annual or biennial (in cold areas) herb. Representative specimen: Dhaka: Ashulia, 15.1.2002, M. Khatun 132. Area of major consumption: Dhaka, also all over the country.

62. B. rapa L., Sp. Pl.: 666 (1753). Vernacular names: Turnip (Eng.), Shalgam (Beng.).

An annual or biannual herb. Representative specimen: Rajshahi: Meherchandi, 13.9.2001, M. Khatun 55. Area of major consumption: Rajshahi district. 
63. Raphanus sativus L., Sp. Pl. 2: 669 (1753). Vernacular names: Raddish (Eng.), Mula (Beng.), Mou-laa (Rakhain).

An annual herb. Representative specimen: Barisal: Barisal Sadar, 11.4.2010, M. Khatun 578. Area of major consumption: Barisal and Jessore districts.

19. Moringaceae Dumortier (1829)

64. Moringa oleifera Lamk., Encycl. 1(2): 398 (1785). Vernacular names: Drumstick Tree, Horseradish Tree (Eng.), Sajna, Sojne (Beng.), Pepan-yuw-maa (Marma).

A medium-sized tree. Representative specimen: Cox's Bazar: Kolatoli, 2.8.2009, M. Khatun 505. Area of major consumption: Cox's Bazar district.

20. Mimosaceae R. Brown (1814)

65. Albizia procera (Roxb.) Benth. in Hook., London J. Bot. 3: 89 (1844). Vernacular names: White Siris (Eng.), Sada Koroi (Beng.), Fonagula (Chakma).

A large, deciduous tree. Representative specimen: Chittagong: Alutila, 8.7.2003, M. Khatun 364. Area of major consumption: Khagrachari district.

\section{Caesalpiniaceae R. Brown (1814)}

66. Bauhinia acuminata L., Sp. Pl. : 375 (1753). Vernacular names: White Bauhinia, Mountain Ebony (Eng.), Shada Kanchon (Beng.), Hingshiara (Garo), Jalong (Khasia).

A large shrub or small tree. Representative specimen: Moulvi Bazar: Madhabkundu, 5.5.2003, M. Khatun 287. Area of major consumption: Moulvi Bazar and Netrakona districts.

67. Caesalpinia digyna Rottler, Neue Schriften Ges. Naturf. Freunde Berlin. 4: 200 (1803). Vernacular names: Kamuno (Marma), Loho (Tanchangya).

A large, straggling, scandent shrub. Representative specimen: Bandarban: Balaghata, 5.3.2012, M. Khatun 621. Area of major consumption: Bandarban district.

68. Cassia fistula L., Sp. Pl.: 377 (1753). Vernacular names: Golden Shower Tree, Purging Cassia (Eng.), Sonalu, Bandar Lati (Beng.), Shumrol (Khasia).

A medium-sized deciduous tree. Representative specimen: Jessore: Hashimpur, 5.1.2004, M. Khatun 389. Area of major consumption: Jessore and Moulvi Bazar districts.

69. Senna obtusifolia (L.) Irwin \& Barneby, Mem. N. Y. Bot. Gard. 35: 252 (1982). Vernacular names: Java Bean (Eng.), Chakunda (Beng.), Dang Geya (Marma).

An erect herb or undershrub. Representative specimen: Bandarban: Lama, 3.4.2011, M. Khatun 615. Area of major consumption: Bandarban district.

70. S. sophera (L.) Roxb., Fl. Ind. 2: 347 (1832). Vernacular names: Pepper-leaved Senna (Eng.), Kalkashunda, Kasundi (Beng.), Eshi Shak (Rakhain).

An undershrub or shrub. Representative specimen: Cox's Bazar: Kolatoli, 2.8.2009, M. Khatun 503. Area of major consumption: Manikganj and Dinajpur districts.

71. S. tora (L.) Roxb., Fl. Ind. 2: 340 (1832). Vernacular names: Sickle Senna (Eng.), Chakunda, Kalkasham (Beng.), Sa Lai Pa (Marma).

An erect foetid herb or undershrub. Representative specimen: Cox's Bazar: Kolatoli, 2.8.2010, M. Khatun 529. Area of major consumption: Rangamati district. 
22. Fabaceae Lindley (1836)

72. Cajanus cajan (L.) Millsp., Publ. Field. Columb. Mus. Bot. Ser. 2: 53 (1900). Vernacular names: Pigeon Pea (Eng.), Arhar (Beng.), Rahar (Santal).

A shrub. Representative specimen: Khulna: Rupdia, 13.9.2007, M. Khatun 443. Area of major consumption: Moulvi Bazar district.

73. Cicer arietinum L., Sp. Pl. 2: 738 (1753). Vernacular names: Chickpea, Bengal Gram (Eng.), Chola, But, Chana (Beng.).

An annual herb. Representative specimen: Natore: Singair, 14.9.2001, M. Khatun 58. Area of major consumption: Natore district.

74. Erythrina stricta Roxb., Fl. Ind. 3: 251 (1832). Vernacular names: Mandar, Teliamandar (Beng.), Kosano (Marma, Tanchangya).

A large, deciduous tree. Representative specimen: Bandarban: Kibukpara, 5.3.2012, M. Khatun 619. Area of major consumption: Bandarban district.

75. Lablab purpureus (L.) Sweet., Hort. Brit. Ed. 1: 481 (1827). Vernacular names: Hyacinth Bean, Lablab (Eng.), Sheem, Urshi, Ushi (Beng.).

A perennial or annual climbing herb. Representative specimen: Cox's Bazar: St. Martin's Island, 18.2.2011, M. Khatun 595. Area of major consumption: Cox's Bazar district.

76. Lathyrus sativus L., Sp. Pl. : 730 (1753). Vernacular names: Grass Pea (Eng.), Khesari (Beng.).

A much branched annual herb. Representative specimen: Natore: Singair, 14.9.2001, M. Khatun 59. Area of major consumption: Rajshahi district.

77. Phaseolus vulgaris L., Sp. Pl. 1: 723 (1753). Vernacular names: Common Bean, Kidney Bean (Eng.), Felong Dal (Marma, Chakma).

A climbing herb. Representative specimen: Bandarban: Bandarban Sadar, 5.3.2012, M. Khatun 620. Area of major consumption: Bandarban district.

78. Pisum sativum L., Sp. Pl.: 727 (1753). Vernacular names: Garden Pea, Pea (Eng.), Motor, Motorshuti (Beng.).

A short-lived, climbing annual herb. Representative specimen: Pirojpur: Nazirpur, 11.4.2010, M. Khatun 583. Area of major consumption: Greater Faridpur district.

79. Sesbania grandiflora (L.) Poir. in Lamk., Encycl. Met. 7: 127 (1806). Vernacular names: Bakful (Beng.), Agasta (Rakhain).

A soft-wooded small tree. Representative specimen: Patuakhali: Kuakata, 23.5.2000, M. Khatun 482. Area of major consumption: Khagrachari and Patuakhali districts.

80. Trigonella foenum-graecum L., Sp. Pl. 3: 777 (1753). Vernacular names: Fenugreek (Eng.), Methi (Beng.).

An annual, robust, aromatic herb. Representative specimen: Rajshahi: Kazla, 13.9.2001, M. Khatun 45. Area of major consumption: Dhaka district.

81. Vigna mungo (L.) Happer. in Kew Bull. 11: 128 (1956). Vernacular names: Black Gram (Eng.), Mashkalai (Beng.), Pee-shee-kanshi-deal (Rakhain).

An annual herb. Representative specimen: Chapai Nawabganj: Moharajpur, 15.9.2001, M. Khatun 71. Area of major consumption: Chapai Nawabganj district. 
23. Onagraceae A. L. de Jussieu (1789)

82. Ludwigia adscendens (L.) Hara, J. Jap. Bot. 28: 290 (1953). Vernacular names: Kesardam (Beng.), Mulsishak (Garo), Gandu-pawn (Rakhain).

An aquatic herb. Representative specimen: Natore: Natore Sadar, 14.9.2001, M. Khatun 60. Area of major consumption: Netrakona district.

24. Melastomataceae A. L. de Jussieu (1789)

83. Osbeckia stellata Buch.-Ham. ex Ker-Gawl., Bot. Reg. 8: 674 (1822). Vernacular names: Star Osbeckia (Eng.), Gaichi (Beng.), Chakum (Khasia).

A shrub. Representative specimen: Moulvi Bazar: Madhabkundu, 3.5.2003, M. Khatun 273. Area of major consumption: Moulvi Bazar district.

25. Euphorbiaceae A. L. de Jussieu (1789)

84. Antidesma acidum Retz., Obs. Bot. 5: 30 (1788). Vernacular names: Indian Laurel (Eng.), Multa (Beng.), Mokhichikra (Tripura).

A large shrub or small tree. Representative specimen: Habiganj: Chunarughat, 6.5.2003, M. Khatun 307. Area of major consumption: Habiganj district.

85. Manihot esculenta Crantz, Inst. 1: 167 (1766). Vernacular names: Cassava (Eng.), Simul-alu (Garo), Kepalli Nolpai (Marma).

A shrub. Representative specimen: Tangail: Madhupur, 18.4.2002, M. Khatun 172. Area of major consumption: Tangail and Sherpur districts.

86. Ricinus communis L., Sp. Pl.: 1007 (1753). Vernacular names: Castor (Eng.), Venna, Rerhi, Bherenda (Beng.), Crusuba (Marma, Tanchangya).

A shrubby or tree-like, somewhat herb. Representative specimen: Bandarban: Bandarban Sadar, 7.3.2012, M. Khatun 617. Area of major consumption: Bandarban district.

26. Vitaceae A. L. de Jussieu (1789)

87. Cissus adnata Roxb., Fl. Ind. ed. Carey 1: 405 (1820). Vernacular names: Alianga-lata, Bhatia-lata (Beng.) Chuka-blei (Tripura).

A slender, woody climber. Representative specimen: Cox’s Bazar: Kolatoli, 2.8.2009, M. Khatun 508. Area of major consumption: Cox's Bazar district.

88. C. assamica (M. Lawson) Craib in Kew Bull.: 31 (1911). Vernacular names: Amasha-pata (Beng.), Gelia Bleli (Tripura).

A large, woody climber. Representative specimen: Moulvi Bazar: Sreemangal, 2.5.2003, M. Khatun 283. Area of major consumption: Moulvi Bazar district.

89. C. elongata Roxb., Fl. Ind. 1: 411 (1832). Vernacular names: Dhemna, Chemna (Beng.).

A large, climbing herb. Representative specimen: Bandarban: Lama, 3.4.2011, M. Khatun 615. Area of major consumption: Bandarban district.

90. C. quadrangularis L., Syst. Nat. ed. 12(2): 124 (1767). Vernacular names: Harjora Lata, Harbhanga Lata (Beng), Marang Gach (Santal), Moi-bhanga Lota (Garo).

A large climber. Representative specimen: Patuakhali: Kalachanpara, 23.5.2008, M. Khatun 495. Area of major consumption: Patuakhali district. 
91. C. repens Lamk., Encycl. Math. Bot. 1: 31 (1783). Vernacular names: Marnaria Pata (Beng.), Marmaria Lata (Koch)

A large, herbaceous climber. Representative specimen: Chittagong: Jamtoli, 8.7.2003, M. Khatun 374. Area of major consumption: Sherpur district.

92. Tetrastigma angustifolium (Roxb.) Planch. in DC., Monogr. Phan. 5: 439 (1887). Vernacular name: Nekung Rubi (Beng.).

A large, glabrous, herbaceous climber. Representative specimen: Rangamati: Kaptai, Digholchari, 7.7.2003, M. Khatun 359. Area of major consumption: Rangamati district.

27. Sapindaceae A. L. de Jussieu (1789)

93. Cardiospermum halicacabum L., Sp. Pl.: 366 (1753). Vernacular names: Balloon Vine, Pigeon's Knee (Eng.), Phutka, Lataphutki (Beng.), Kataboksa Shak (Chakma).

An annual or perennial climbing herb. Representative specimen: Rangamati: Kaptai, Digholchari, 7.7.2003, M. Khatun 377. Area of major consumption: Rangamati district.

\section{Anacardiaceae Lindley (1830)}

94. Mangifera indica L., Sp. Pl.: 200 (1753). Vernacular names: Mango (Eng.), Aam (Beng.), Kharai (Khasia), Tharaapang (Rakhain).

A large tree. Representative specimen: Moulvi Bazar: Adampur, 3.5.2003, M. Khatun 267. Area of major consumption: Moulvi Bazar district.

95. Spondias pinnata (L. f.) Kurz, Pegu. Rep.: 44 (1875). Vernacular names: Hog Plum (Eng.), Deshi-amra (Beng.), Thai-toui (Tripura), Soh-awla (Khasia).

A medium-sized to large tree. Representative specimen: Habiganj: Chunarughat, 6.5.2003, M. Khatun 313. Area of major consumption: Moulvi Bazar district.

29. Meliaceae A. L. de Jussieu (1789).

96. Azadirachta indica A. Juss. in Mem. Mus. Nat. Hist. Paris 19: 221, t. 13 (1830). Vernacular names: Margosa Tree, Neem Tree (Eng.), Neem (Beng.), Hoppa (Rakhain).

A medium-sized to large tree. Representative specimen: Patuakhali: Kuakata, 24.5.2008, M. Khatun 486. Area of major consumption: Patuakhali district.

30. Rutaceae A. L. de Jussieu (1789)

97. Aegle marmelos (L.) Corr., Trans. Linn. Soc. Lond. 5: 223 (1800). Vernacular names: Bengal Quince (Eng.), Bel (Beng.), War-e-si-apang (Marma).

A deciduous tree. Representative specimen: Bandarban: Balaghata, 5.3.2012, M. Khatun 616. Area of major consumption: Bandarban and Khagrachari districts.

98. Clausena excavata Burm. f., Fl. Ind.: 87, t. 29, 2 (1768). Vernacular names: Clausena (Eng.), Pan Karpur (Beng.), Pankauri (Chakma).

An aromatic shrub. Representative specimen: Chittagong: Sitakunda, 7.7.2003, M. Khatun 285. Area of major consumption: Rangamati district.

99. Zanthoxylum rhetsa (Roxb.) DC., Prodr. 1: 728 (1824). Vernacular names: Indian Ivy-rue (Eng.), Bajna, Kantahorina, Tambol (Beng.), Khazai (Garo).

A medium-sized, deciduous tree. Representative specimen: Tangail: Madhupur, 18.4.2002, M. Khatun 178. Area of major consumption: Tangail and Netrakona districts. 
31. Oxalidaceae R. Brown (1817)

100. Oxalis corniculata L., Sp. Pl.: 435 (1753). Vernacular names: Indian Sorrel (Eng.), Amrul, Amrul Shak (Beng.), Amila Pata (Chakma, Khasia).

A small perennial herb. Representative specimen: Rangamati: Kaptai, Digholchari, 7.7.2003, M. Khatun 348. Area of major consumption: Rangamati district.

\section{Apiaceae Lindley (1836)}

101. Centella asiatica (L.) Urban in Mart. \& Eichler, Fl. Brasil. 11 (1): 287 (1879). Vernacular names: Indian Pennywort (Eng.), Thankuni, Brahmabuti (Beng.).

A perennial herb. Representative specimen: Dhaka: Bocila, 15.1.2002, M. Khatun 167. Area of major consumption: Dhaka district.

102. Coriandrum sativum L., Sp. Pl. 1: 256 (1753). Vernacular names: Coriander (Eng.), Dhonay, Dhonia (Beng.).

An annual herb. Representative specimen: Chittagong: Adampur, 6.7.2003, M. Khatun 372. Area of major consumption: Dhaka district.

103. Eryngium foetidum L., Sp. Pl. 1: 232 (1753). Vernacular names: Wild Coriander (Eng.), Mysapagur (Chakma).

An erect, biennial herb. Representative specimen: Rangamati: Digholchari, 7.7.2003, M. Khatun 351. Area of major consumption: Rangamati district.

104. Foeniculum vulgare (L.) Miller, Gard. Diet. ed. 8, no. 1 (1768). Vernacular names: Funnel (Eng.), Pan-mohuri (Beng.), Moroi (Marma)

A robust, aromatic herb. Representative specimen: Bandarban: Bandarban Sadar, 7.3.2012, M. Khatun 616. Area of major consumption: Bandarban district.

105. Hydrocotyle sibthorpioides Lamk., Enc. 3: 153 (1789). Vernacular names: Lawn Marsh Pennywort (Eng.), Sakumubakla (Marma).

A perennial, slender herb. Representative specimen: Khagrachari: Bottoli, 6.7.2003, M. Khatun 339. Area of major consumption: Rangamati district.

106. Oenanthe benghalensis (Roxb.) Kurz, J. Asiat. Soc. Beng. 2: 115 (1877). Vernacular names: Water Celery (Eng.), Bandhunia (Khasia).

A perennial, glabrous herb. Representative specimen: Rangamati: Kaptai, Bangchari, 6.7.2003, M. Khatun 343. Area of major consumption: Rangamati district.

107. O. javanica (Blume) DC., Prodr. 4: 138 (1830). Vernacular names: Water Dropwort, Java Waterdropwort (Eng.), Pan-turasi (Beng.), Branju (Rakhain).

A perennial herb. Representative specimen: Patuakhali: Kalachandpara, 23.5.2008, M. Khatun 491. Area of major consumption: Patuakhali district.

108. Trachyspermum ammi (L.) Sprague, Bull. Misc. Inform. Kew. 1929: 228 (1929). Vernacular names: Ajowan Caraway (Eng.), Jawan (Beng.), Fuchi Shak (Chakma, Marma).

An annual herb. Representative specimen: Khagrachari: Golabari, 6.7.2003, M. Khatun 326. Area of major consumption: Khagrachari district.

109. T. roxburghianum (DC.) H. Wolff in Engl., Pfl. Umbellif. Apioid-Ammin.: 129 (1927). Vernacular names: Radhuni, Chanu (Beng.), Rajani (Marma). 
An annual, aromatic herb. Representative specimen: Dhaka: Bosila, 15.10.2002, M. Khatun 233. Area of major consumption: Bandarban district.

33. Solanaceae A. L. de Jussieu (1789)

110. Capsicum frutescens L., Sp. Pl.: 189 (1753). Vernacular names: Spur Pepper, Cayenne Pepper (Eng.), Kacha Morich (Beng.).

A herb. Representative specimen: Patuakhali: Patuakhali Sadar, 23.5.2008, M. Khatun 493. Area of major consumption: Patuakhali district.

111. Physalis angulata L., Sp. Pl. : 183 (1753). Vernacular names: Hogweed, Balloon Cherry (Eng.), Potka (Beng.), Ambichok (Garo).

An annual, much branched herb. Representative specimen: Pabna: Thanapara, 5.3.2007, M. Khatun 467. Area of major consumption: Pabna district.

112. Solanum americanum Mill., Gard. Dict. ed. 8, No. 5 (1768). Vernacular names: Glossy Nightshade (Eng.), Titbegun (Beng.).

An erect annual herb. Representative specimen: Habiganj: Chunarughat, 6.5.2003, M. Khatun 316. Area of major consumption: Habiganj district.

113. S. tuberosum L., Sp. Pl.: 185 (1753). Vernacular names: Potato (Eng.), Alu, Gol Alu (Beng.), Mraa-u-shey (Rakhain).

A viscoid herb. Representative specimen: Munshiganj: Gozaria, 18.1.2010, M. Khatun 566. Area of major consumption: Cox's Bazar district.

114. S. villosum Mill., Gard. Dict. ed. 8, no. 2 (1768). Vernacular names: Orange Nightshade (Eng.), Titbegun, Kakmachi (Beng.), Kha-rey-je-key (Rakhain).

An annual herb. Representative specimen: Patuakhali: Keranipara, 23.5.2008, M. Khatun 490. Area of major consumption: Chapai Nawabganj district.

34. Convolvulaceae A. L. de Jussieu (1789)

115. Hewittia sublobata (L. f.) O. Kuntze, Rev. Gen. Pl. 2 : 441 (1891). Vernacular name: Dhudla Shak (Beng).

A twining perennial herb. Representative specimen: Rajbari: Salki, 12.3.2006, M. Khatun 403. Area of major consumption: Rajbari and Feni districts.

116. Ipomoea aquatica Forssk., Fl. Aeg.-Arab. : 44 (1755). Vernacular names: Water Spinach (Eng.), Kalmi Shak (Beng.).

An aquatic herb. Representative specimen: Gopalganj: Digholia, 3.3.2007, M. Khatun 424. Area of major consumption: Gopalganj district.

117. I. batatus (L.) Lamk., Tabl. Encycl. 1: 465 (1791). Vernacular names: Sweet Potato (Eng.), Misti Alu, Ranga Alu (Beng.).

A perennial herb. Representative specimen: Dhaka: Diabari, 15.1.2002, M. Khatun 134. Area of major consumption: Dhaka district.

118. Operculina turpethum (L.) S. Manso., Enum. Subst. Bras.: 16 (1836). Vernacular names: Turpeth Root (Eng.), Dudh Kalmi (Beng.).

A glabrous twiner. Representative specimen: Patuakhali: Kolapara, 23.5.2008, M. Khatun 479. Area of major consumption: Gazipur and Patuakhali districts. 
35. Verbenaceae Jaume St.- Hilaire (1805)

119. Clerodendrum inerme (L.) Gaertn., Fruct. Sem. Pl. 1: 271 (1788). Vernacular names: Glory Bower (Eng.), Banjui, Batraj, Koklata (Beng.), Jarems (Khasia).

An erect to scandent shrub. Representative specimen: Moulvi Bazar: Madhabpunji, 4.5.2003, M. Khatun 282. Area of major consumption: Moulvi Bazar district.

120. Premna benghalensis C. B. Clarke in Hook. f., Fl. Brit. Ind. 4: 577 (1885). Vernacular names: Pakhirhar (Beng.), Koya Jarul (Khasia).

A medium-sized evergreen tree. Representative specimen: Habiganj: Chunarughat, 6.5.2003, M. Khatun 317. Area of major consumption: Habiganj district.

121. P. esculenta Roxb., Fl. Ind. ed. 2, 3: 81 (1832). Vernacular names: Lalong, Lalana (Beng.), Gun-duri, Darkakha (Khasia).

A small shrub. Representative specimen: Habiganj: Chunarughat, Kalenga, 6.5.2003, M. Khatun 312. Area of major consumption: Habiganj district.

122. P. mucronata Roxb., Fl. Ind. ed. 2, 3: 635 (1832). Vernacular name: Khatamuri (Koch).

A shrub or small tree. Representative specimen: Sherpur: Runctia, Kochpara, 1.11.2009, M. Khatun 531. Area of major consumption: Sherpur district.

123. P. obtusifolia R. Br., Prod. Fl. Nov. Holl. 1: 512 (1810). Vernacular names: Gambari, Bhuttsirabi (Beng.), Lalom Pata (Khasia, Chakma).

A shrub to small evergreen tree. Representative specimen: Khagrachari: Golabari, 6.7.2003, M. Khatun 318. Area of major consumption: Khagrachari district.

\section{Lamiaceae Lindley (1836).}

124. Ajuga macrosperma Wall. ex Benth. in Wall., Pl. As. Rar. 1: 58 (1830). Vernacular names: Sabarang (Chakma, Marma).

A herb. Representative specimen: Khagrachari: Golabari, 6.7.2003, M. Khatun 337. Area of major consumption: Khagrachari and Rangamati districts.

125. Leucas aspera (Willd.) Link, Enum. Hort. Berol. 2: 113 (1822). Vernacular names: Dondokalosh (Beng.), Dong-Ke-La (Coach).

A stout, erect or diffuse, annual herb. Representative specimen: Bandarban: Lama, 3.4.2011, M. Khatun 609. Area of major consumption: Chittagong district.

126. L. cephalotes (Roth.) Spreng., Syst. 2: 743 (1825). Vernacular names: Bara Kalkus (Beng.), Thaelsi (Garo).

A stout, erect, annual herb. Representative specimen:: Netrakona: Bijoypur, 8.10.2000, M. Khatun 05. Area of major consumption: Netrakona district.

127. Ocimum americanum L., Cent. Pl. 1: 15 (1755). Vernacular names: Rosary Ocimum (Eng.), Tulshi (Beng.), Bontulsi (Coach).

An erect, annual, aromatic herb. Representative specimen: Rangamati: Kaptai, Koblachara, 7.7.2003, M. Khatun 347. Area of major consumption: Rangamati district.

128. Pogostemon benghalensis (Burm. f.) O. Kuntze, Rev. Gen. Pl. 2: 529 (1891). Vernacular name: Lomboi Shak (Chakma, Marma, Khasia). 
An erect, stout, aromatic undershrub. Representative specimen: Rangamati: Kaptai, Digholchari, 6.7.2003, M. Khatun 338. Area of major consumption: Rangamati district.

37. Scrophulariaceae A. L. de Jussieu (1789)

129. Bacopa monnieri (L.) Pennell in Pflanzenfam. 4(3b): 77 (1891). Vernacular names: Water Hyssop (Eng.), Brammi, Brammi Shak, Dupkalmini (Beng.)

An annual herb. Representative specimen: Cox's Bazar: St. Martin's Island, 18.2.2011, M. Khatun 596. Area of major consumption: Patuakhali and Cox's Bazar districts.

130. Scoparia dulcis L., Sp. Pl.: 116 (1753). Vernacular names: Goat Weed (Eng.), Bondhone (Beng.), Shamgaldak (Garo).

An erect, much branched, perennial herb. Representative specimen: Dinajpur: Mukundupur, 16.9.2001, M. Khatun 87. Area of major consumption: Dinajpur district.

38. Acanthaceae A. L. de Jussieu (1789)

131. Hygrophila polysperma (Roxb.) T. Anders., Journ. Linn. Soc. Bot. 9: 456 (1867). Vernacular names: Dwarf Hygrophila (Eng.), Puinnya Shak (Beng.), Puinna Shak (Rakhain).

A small, much branched herb. Representative specimen: Patuakhali: Kalapara, 23.5.2008, M. Khatun 496. Area of major consumption: Patuakhali district.

132. H. schulli (Buch.-Ham.) M. R. \& S. N. Almeida, Journ. Bomb. Nat. Hist. Soc. 83 (Suppl.): 221 (1986). Vernacular names: Star Thorn (Eng.), Talmakhna, Kulekhara (Beng.).

An annual, erect herb. Representative specimen: Jhalakathi: Jhalakathi proper, 11.4.2010, M. Khatun 577. Area of major consumption: Barisal and Rajbari districts.

133. Nelsonia canescens (Lamk.) Spreng., Syst. 1 : 42 (1824). Vernacular names: Paramul (Beng.), Khaia Shak (Khasia).

A trailing or diffuse herb. Representative specimen: Habiganj: Chunarughat, 6.5.2003, M. Khatun 302. Area of major consumption: Habiganj district.

134. Ruellia tuberosa L., Sp. Pl.: 635 (1753). Vernacular names: Blue Bell (Eng.), Chatpotey (Beng.), Charasak (Khasia).

A perennial erect herb. Representative specimen: Moulvi Bazar: Lawacherra, 2.5.2003, M. Khatun 249. Area of major consumption: Habiganj and Moulvi Bazar districts.

39. Bignoniaceae A. L. de Jussieu (1789)

135. Oroxylum indicum (L.) Kurz, For. Fl. Brit. Burm. 2: 237 (1877). Vernacular names: Midnight Horror (Eng.), Kanaidingi (Beng.), Kharam-sha-bawn (Rakhain), Fona-gulogach (Chakma).

A medium-sized tree. Representative specimen: Patuakhali: Kuakata, 24.5.2008, M. Khatun 478. Area of major consumption: Patuakhali and Rangamati districts.

40. Sphenocleaceae Lindly (1829)

136. Sphenoclea zeylanica Gaertn., Fruct. Sem. Pl. 1: 113 (1788). Vernacular names: Jhill Mirich (Beng.), Vui Shak (Chakma), Radai (Mog).

A robust herb. Representative specimen: Rangamati: Kaptai, Debachari, 7.7.2003, M. Khatun 356. Area of major consumption: Khagrachari district. 
41. Rubiaceae A. L. de Jussieu (1789)

137. Hedyotis corymbosa (L.) Lamk., Tab. Encycl. 1: 272 (1791). Vernacular names: Khetpapra, Panki (Beng.), Dimatita (Marma).

An annual, diffuse or prostrate herb. Representative specimen: Dinajpur: Mukundopur, 16.9.2001, M. Khatun 79. Area of major consumption: Dinajpur district.

138. Morinda citrifolia L., Sp. Pl.: 176 (1753). Vernacular names: Ach, Banach, Tufania (Beng.), Ken-thug-blag, Chirasak (Khasia).

A shrub or small tree. Representative specimen: Cox's Bazar: Kolatoli, 2.8.2009, M. Khatun 506. Area of major consumption: Chittagong district.

139. Paederia foetida L., Mant. 1: 52 (1767). Vernacular names: Gandha Bhaduli, Badali (Beng.), Padbaj Ludi (Chakma).

A twining, glabrous shrub. Representative specimen: Netrakona: Polastola, 8.10.2001, M. Khatun 14. Area of major consumption: Netrakona district.

140. Spermacoce articularis L. f., Sppl. Pl: 119 (1782). Vernacular names: Horinshing, Usni (Santal).

A procumbent, perennial, mat forming herb. Representative specimen: Dinajpur: Noyabad, 16.9.2001, M. Khatun 86. Area of major consumption: Dinajpur district.

141. S. latifolia Aublet, Hist. Pl. Guiane Fr. 1: 55, t. 194 (1755). Vernacular name: Ghuiojhil Shak (Tanchangya).

A prostrate or decumbent herb. Representative specimen: Bandarban: Lama, 3.4.2011, M. Khatun 612. Area of major consumption: Bandarban district.

142. S. stricta L. f., Suppl. Pl.: 120 (1781). Vernacular names: Narkel Jhuri Shak (Rakhain), Mijlick (Chakma).

An erect or rarely prostrate, annual herb. Representative specimen: Patuakhali: Keranipara, 23.5.2008, M. Khatun 492. Area of major consumption: Patuakhali and Sylhet districts.

42. Caprifoliaceae A. L. de Jussieu (1789)

143. Sambucus javanica Reinw. ex Blume, Bijdr.: 657 (1826). Venacular names: Javanese Elder (Eng.), Hoklati (Beng.), Maytraba (Chakma).

A large shrub or small tree. Representative specimen: Rangamati: Rangamati sadar, 10.7.2003, M. Khatun 333. Area of major consumption: Bandarban and Rangamati districts.

43. Asteraceae Dumortier (1822)

144. Ageratum conyzoides L., Sp. Pl.: 839 (1753). Vernacular names: Tropical White Weed (Eng.), Ozone Shak (Chakma), Mukri (Tripura), Hinor (Khasia).

An annual herb. Representative specimen: Khagrachari: Bot-toli, 6.7.2003, M. Khatun 323. Area of major consumption: Khagrachari district.

145. Blumea lacera (Burm. f. ) DC. in Wight, Contr. Bot. Ind.: 14 (1834). Vernacular names: Barakukshim, Kukurshunga, Kuksung (Beng.), Leikhamal (Manipuri).

An annual aromatic herb. Representative specimen: Moulvi Bazar: Komolganj, 3.5.2003, M. Khatun 271. Area of major consumption: Feni and Moulvi Bazar districts. 
146. Elephantopus scaber L., Sp. Pl.: 814 (1753). Vernacular names: Banmula (Beng.), Gejia Shak (Koch).

A perennial herb. Representative specimen: Noakhali: Begumganj, 7.5.2003, M. Khatun 321. Area of major consumption: Noakhali district.

147. Emilia sonchifolia (L.) DC. in Wight, Contrib. : 24 (1834). Vernacular names: Lilac Tassel Flower (Eng.), Mechitra, Sadusi (Beng.), Miam Shak (Chakma).

An annual branched herb. Representative specimen: Khagrachari: Dokkhin Golabari, 8.7.2003, M. Khatun 376. Area of major consumption: Khagrachari district.

148. Enhydra fluctuans Lour., Fl. Chchinch.: 511 (1790). Vernacular names: Helencha, Hingcha, Harhach (Beng.).

An annual, aquatic herb. Representative specimen: Gopalganj: Tungipara, 4.3.2007, M. Khatun 299. Area of major consumption: Gopalganj district.

149. Lactuca scariola L. var. sativa Hook. f., Fl. Brit. Ind. 3 : 404 (1881). Vernacular names: Garden Lettuce (Eng.), Latus Pata (Beng.).

An annual or biennial herb. Representative specimen: Rajshahi: Meharchandi, 13.9.2001, M. Khatun 36. Area of major consumption: Dhaka district.

150. Spilanthes calva DC. in Wight, Contrib. : 19 (1834). Vernacular names: Paracress (Eng.), Surfa, Kannya (Beng.), Marhatitiga (Marma)

An annual herb. Representative specimen: Khagrachari: Shomoboy market, 6.7.2003, M. Khatun 340. Area of major consumption: Rangamati district.

151. Synedrella nodiflora (L.) Gaertn., Fruct. 2: 456, t. 171 (1791). Vernacular name: Hamfui (Marma, Chakma).

An annual pubescent herb. Representative specimen: Khagrachari: Pouromarket, 6.7.2003, M. Khatun 344. Area of major consumption: Khagrachari district.

152. Vernonia cinerea (L.) Less., Linnaea 4 (1): 291 (1829). Vernacular names: Little Ironweed (Eng.), Kuksim (Beng.), Kalojira (Garo).

An erect, annual or perennial herb. Representative specimen: Dinajpur: Noiyabad, 16.9.2001, M. Khatun 98. Area of major consumption: Netrakona district.

153. Xanthium indicum Koen. ex Roxb., Fl. Ind. 3: 601 (1832). Vernacular names: Rough Cocklebur (Eng.), Ghagra, Ban-okra (Beng.), Baksala (Hajong).

An annual herb. Representative specimen: Munshiganj: Mahakali, 18.1.2010, M. Khatun 568. Area of major consumption: Barisal, Faridpur, Madaripur, Rajshahi and Chittagong districts.

Note: Seedlings of this species are highly poisonous, thus should never be plucked for vegetables in this stage.

\section{LILIOPSIDA}

1. Arecaceae C.H. Schultz-Schultzen. (1832)

154. Calamus tenuis Roxb., Fl. Ind. 3: 780 (1832). Vernacular names: Rattan (Eng.), Bet (Beng.), Khring (Marma).

A thicket forming climber. Representative specimen: Khagrachari: Bot-toli, 6.7.2003, M. Khatun 330. Area of major consumption: Khagrachari district. 
2. Araceae A.L. de Jussieu (1789)

155. Amorphophallus bulbifer (Roxb.) Blume, Rumph. 1: 148 (1847). Vernacular names: Voodoo Lily (Eng.), Jongli Ol (Beng.), Chungmuru (Garo).

A herb. Representative specimen: Sherpur: Runctia, 31.10.2009, M. Khatun 539. Area of major consumption: Sherpur and Moulvi Bazar districts.

156. A. nepalensis (Wall.) Bogner \& Mayo, Aroideana 8(1): 19 (1985). Vernacular names: Khar Kochu (Tripuri), Dadonga (Koch).

A tuberous herb. Representative specimen: Mymensingh: Haluaghat, 6.10.2009, M. Khatun 115. Area of major consumption: Mymensingh district.

157. Colocasia esculenta (L.) Schott in Schott \& Endl., Melet. Bot. 1: 18 (1832). Vernacular names: Taro, Coco-yam (Eng.), Kachu (Beng.).

A perennial herb. Representative specimen: Jessore: Monirampur, 5.1.2004, M. Khatun 382. Area of major consumption: Jessore and Dhaka districts.

158. C. gigantea (Blume) Hook. f., Fl. Brit. Ind. 6: 524 (1893). Vernacular names: Giant Elephant Ear (Eng.), Salad Kachu (Beng.), Chinjapang (Khasia).

A perennial herb. Representative specimen: Khagrachari: Silchari, 8.7.2003, M. Khatun 334. Area of major consumption: Khagrachari and Sylhet districts.

159. Homalomena aromatica (Roxb. ex Sims.) Schott in Schott \& Endl., Melet. Bot. 1: 20 (1832). Vernacular names: Bonkachu (Beng.), Kachu Gondhobi (Khasia), Chikon Shak (Marma).

A perennial herb. Representative specimen: Moulvi Bazar: Adampur bit, 3.5.2003, M. Khatun 268. Area of major consumption: Moulvi Bazar district.

160. Lasia spinosa (L.) Thwait., Enum. Pl. Zeyl. 1: 336 (1864). Vernacular names: Kanta Kachu (Beng.), Bonadia (Garo).

A perennial, stout herb. Representative specimen: Netrakona: Durgapur, 8.10.2000, M. Khatun 04. Area of major consumption: Netrakona and Habiganj districts.

161. Typhonium trilobatum (L.) Schott, Wien. Zeitschr. 3: 72 (1829). Vernacular names: Ghetkachu, Ghekul (Beng.), Kharkon (Santal).

A small, tuberous, terrestrial herb. Representative specimen: Gazipur: Rajendrapur, 4.12.2009, M. Khatun 554. Representative specimen: Dhaka and Gazipur districts.

162. Xanthosoma sagittifolium (L.) Schott in Schott \& Endl., Melet. Bot.: 19 (1832) ('sagittaefolium'). Vernacular names: Tannia, Tanier (Eng.), Mukhikachu (Beng.).

A perennial herb. Representative specimen: Rajbari: Salmara, 12.3.2006, M. Khatun 401. Area of major consumption: Faridpur district.

163. X. violaceum Schott, Oesterr. Bot. Wochenbl. 3: 370 (1853). Vernacular names: Blue Taro, Purple-stem Taro (Eng.), Dudhkachu (Beng.).

A perennial herb. Representative specimen: Jessore: Hashimpur, 5.1.2004, M. Khatun 385. Area of major consumption: Jessore district.

3. Commelinaceae R. Brown (1810)

164. Commelina benghalensis L., Sp. Pl.: 41 (1753). Vernacular names: Blue Commelina, Bengal Dayflower (Eng.), Kanchira (Beng.), Piachara (Chakma). 
A small herb. Representative specimen: Chapai Nawabganj: Moharajpur, 12.9.2001, M. Khatun 66. Area of major consumption: Chapai Nawabganj district.

4. Poaceae Barnhart (1895)

165. Melocanna baccifera (Roxb.) Kurz, Prelim. Rep. For. Veg. Pegu, App. B.: 94 (1875). Vernacular names: Berry Bamboo (Eng.), Bajali Muli, Tarai (Beng.), Wa-thui, Muiya (Chakma, Marma, Khasia).

A diffusely clumped, sympodial bamboo. Representative specimen: Khagrachari: Bot-toli, 6.7.2003, M. Khatun 341. Area of major consumption: Chittagong Hill Tracts.

\section{Zingiberaceae Lindley (1835)}

166. Curcuma longa L., Sp. Pl. 1: 2 (1753). Vernacular names: Turmeric (Eng.), Halud, Haldi (Beng.).

A rhizomatous herb. Representative specimen: Cox’s Bazar: Kolatoli, 2.8.2009, M. Khatun 513. Area of major consumption: Cox's Bazar district.

167. Globba marantina L., Mant. Alt. : 170 (1771). Vernacular names: Yellow Dancing Girl (Eng.), Holi Shak (Khasia).

A small, annual herb. Representative specimen: Rangamati: Kaptai, Bangchari, 7.7.2003, M. Khatun 357. Area of major consumption: Khagrachari and Rangamati districts.

168. Zingiber officinale Rosc., Trans. Linn. Soc. Lond. 8: 348 (1807). Vernacular names: Ginger (Eng.), Ada (Beng.), Shaen-pang (Rakhain).

A small, rhizomatous herb. Representative specimen: Cox's Bazar: Kolatoli pahar, 2.8.2009, M. Khatun 501. Area of major consumption: Cox’s Bazar district.

\section{Pontederiaceae Kunth (1816)}

169. Monochoria hastata (L.) Solms in A. DC., Monogr. Phaner. 4: 523 (1883). Vernacular names: Arrowleaf False Pickereweed (Eng.), Baranukha (Beng.), Chichir (Garo), Projukti Shak (Tripura).

A perennial, robust herb. Representative specimen: Netrakona: Bijoypur, 8.10.2001, M. Khatun 13. Area of major consumption: Netrakona district.

170. M. vaginalis (Burm. f.) Presl, Rel. Haenk. 1: 128 (1827). Vernacular names: Heartshape False Pickereweed (Eng.), Nukha (Beng.), Kusrisha (Khasia).

A slender, perennial herb. Representative specimen: Habiganj: Chunarughat, 6.5.2003, M. Khatun 314. Area of major consumption: Khagrachari and Moulvi Bazar districts.

7. Liliaceae A.L. de Jussieu (1789)

171. Allium cepa L., Sp. Pl. ed.1: 300 (1753). Vernacular names: Onion (Eng.), Piaz (Beng.). An annual herb. Representative specimen: Jessore: Hashimpur, 5.7.2004, M. Khatun 394. Area of major consumption: Dhaka district.

172. A. sativum L., Sp. Pl. 1: 297 (1753). Vernacular names: Garlic (Eng.), Rashun (Beng.). An erect herb. Representative specimen: Rajbari: Salmara, 8.2.2008, M. Khatun 417. Area of major consumption: Rajbari district. 
8. Dioscoriaceae R. Brown (1810)

173. Dioscorea pentaphylla L., Sp. Pl.: 1032 (1753). Vernacular names: Five-leaf Yam (Eng.), Jhum Alu, Kanta Alu (Beng.), Khaiamor (Mog), Patil Alu (Coach, Rajbonshi).

A twining herb. Representative specimen: Bandarban: Lama, 3.4.2011, M. Khatun 607. Area of major consumption: Gazipur and Bandarban districts.

\section{PTERIDOPHYTA}

1. Angiopteridaceae Fee ex Bonner (1867)

174. Angiopteris evecta (Forst.) Hoffm., Comm. Soc. Reg. Gott. 12: 29, t. 5 (1796). Vernacular names: King Fern (Eng.), Dhekia Shak (Beng.), Siblu (Marma).

A large, semi-erect tree fern. Representative specimen: Bandarban: Lama, 3.4.2011, M. Khatun 616. Area of major consumption: Bandarban and Cox's Bazar districts.

\section{Athyriaceae Pichi Sermolli (1970)}

175. Diplazium esculentum (Retz.) Sw., Schrad. J. 1801 (2): 312 (1803). Vernacular names: Edible Fern (Eng.), Dhekia Shak (Beng.), Teria Shak (Tripuri).

A terrestrial fern. Representative specimen: Habiganj: Rema-Kalenga, 6.5.2003, M. Khatun 303. Area of major consumption: Chittagong and Sylhet districts.

176. D. polypodioides Bl., En. Pl. Jav.: 194 (1828). Vernacular names: Fern (Eng.), Dhekia (Beng.).

A fern. Representative specimen: Chittagong: Bangchari, 8.7.2003, M. Khatun 361. Area of major consumption: Chittagong and Mymensingh districts.

\section{Blechnaceae (Presl) Copel. (1947)}

177. Blechnum orientale L., Sp. Pl. 2: 1077 (1753). Vernacular name: Boro Dhekia Shak (Garo). A large, terrestrial fern. Representative specimen: Cox's Bazar: Himchari, 2.8.2009, M. Khatun 517. Area of major consumption: Mymensingh and Cox’s Bazar districts.

\section{Dennstaedtiaceae Pichi Sermolli $(1970,1977)$}

178. Microlepia strigosa (Thunb.) Presl, Epim.: 95 (1849). Vernacular names: Lacy Fern (Eng.), Fita Dhekia (Beng.), Dheki Shak (Coach and Garo).

A tufted fern. Representative specimen: Sylhet: Satchari forest, 17.5.2005, Momtaz Mahal Mirza 526 (DACB). Area of major consumption: Habiganj and Sylhet districts.

\section{Helminthostachyaceae Ching (1941)}

179. Helminthostachys zeylanica (L.) Hook., Gen. Fil.: t. : 47 (1840). Vernacular names: Fern (Eng.), Shada Dhekia (Garo).

A terrestrial fern. Representative specimen: Tangail: Pirgacha, 18.4.2002, M. Khatun 173. Area of major consumption: Mymensingh district.

\section{Marsileaceae Mirbel (1802)}

180. Marsilea minuta (L.) Mant.: 308 (1771). Vernacular names: Marshy Fern (Eng.), Susni Shak (Beng.).

A small fern-allies. Representative specimen: Dinajpur: Noyabad, 16.9.2001, M. Khatun 84. Area of major consumption: Natore and Dinajpur districts. 
181. M. quadrifolia L., Sp. Pl. 2: 1099 (1753). Vernacular names: Water Clover (Eng.), Susni Shak (Beng.).

A fern-allies. Representative specimen: Rajshahi: Godagari, 13.9.2001, M. Khatun 46. Area of major consumption: Rajshahi district.

7. Ophioglossaceae (R. Br.) Agardh (1882)

182. Ophioglossum reticulatum L., Sp. Pl. 2: 1063 (1753). Vernacular names: Adder’s Tongue (Eng.), Sharpa Jihba (Beng.).

A terrestrial fern. Representative specimen: Mymensingh: Valukapara, 6.10.2001, M. Khatun 119. Area of major consumption: Mymensingh district.

\section{Parkeriaceae Hook. (1825)}

183. Ceratopteris pteridoides (Hook.) Hiern., Bot. Jahrb. 34: 561 (1905). Vernacular names: Aquatic Fern (Eng.), Pani Fern (Beng.), Pani Dhekia Shak (Garo).

An aquatic fern. Representative specimen: No specimen was collected by the author, but this species has been reported to be used as leafy vegetable by Sarker and Hossain (2009). Area of major consumption: Mymensingh district.

184. C. thalictroides (L.) Brongn., Bull. Soc. Phil. 1821: 186 (1822). Vernacular names: Water Fern (Eng.), Pani Dhekia (Beng.), Keng Khah (Garo).

An aquatic fern. Representative specimen: Bandarban: Lama, 3.4.2011, M. Khatun 610. Area of major consumption: Bandarban district.

\section{Stenochlaenaceae Ching (1970)}

185. Stenochlaena palustris (Burm. f.) Bedd., Ferns Brit. India (Suppl.) : 26 (1876). Vernacular names: Climbing Fern (Eng.), Lata Dhekia (Beng.).

A climbing terrestrial fern. Representative specimen: Dacca: Bander, 17.8.1941, S. K. Sen and Atul 638 (DUSH). Area of major consumption: Chittagong and Dhaka districts.

\section{Thelypteridaceae Ching (1970)}

186. Ampelopteris prolifera (Retz.) Copel., Gen. Fil : 144 (1947). Vernacular names: Walking Fern (Eng.), Dhekia Shak (Beng.).

A creeping pteridophyte. Representative specimen: Chittagong: Foy's lake, 12.7.2004, Momtaz Mahal Mirza 421 (DACB). Area of major consumption: Mymensingh and Chittagong districts.

Taxonomic study on the leafy vegetables is the first in its nature in Bangladesh. The present study shows that Amaranthaceae is the largest family in Magnoliopsida represented by 14 species followed by Asteraceae, Cucurbitaceae and Fabaceae comprising 10 species each. In Liliopsida, Araceae stands the highest position with 9 species followed by Zingiberaceae with 3 species. Out of 10 families of pteridophytes the families Athyriaceae, Marsileaceae and Parkeriaceae possess 2 species each. Twenty seven familes are represented by a single species used as leafy vegetables of which 20 families belong to angiosperms and 7 families to pteridophytes. Fifteen largest families of leafy vegetables reported to be found in Bangladesh are shown in Fig. 1. 


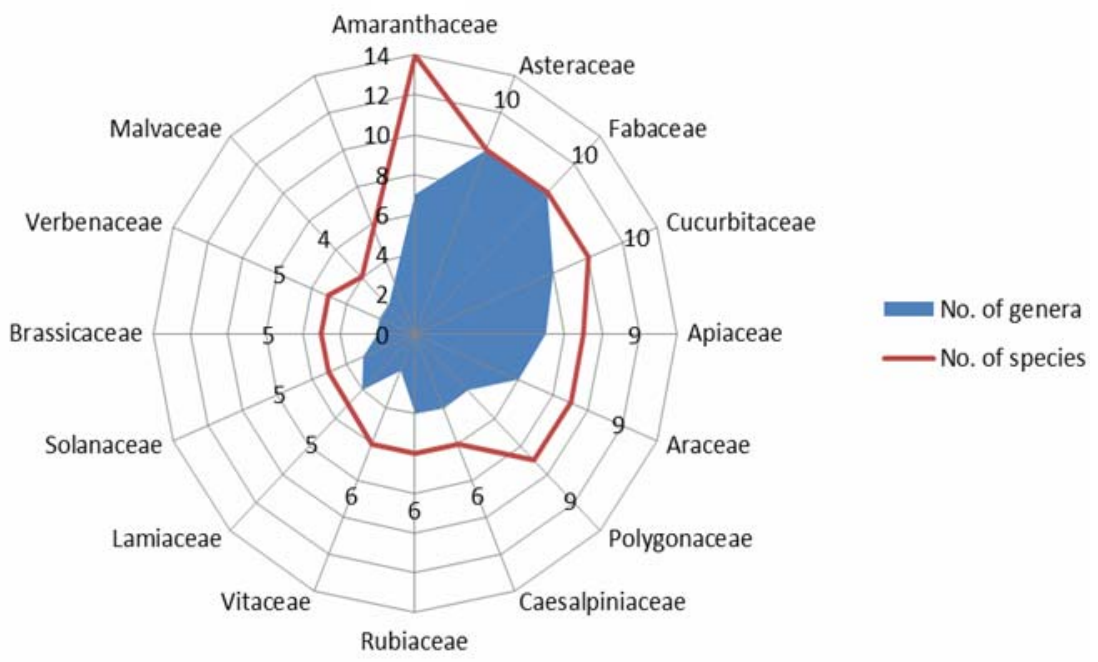

Fig. 1. Radder diagram showing 15 largest families of leafy vegetables in Bangladesh.

The study reveals the identification of 61 newly documented leafy vegetables for Bangladesh (Table 2). The majority of these newly documented leafy vegetables are consumed by the people of the hilly areas, especially in the Chittagong Hill Tracts and greater Sylhet district.

Out of 186 leafy vegetables recorded for Bangladesh, 140 taxa are wild and 46 are cultivated. Among the cultivated ones, 16 taxa are cultivated only for leafy vegetables, viz., Amaranthus tricolor, A. viridis, Basella alba, Benincasa hispida, Brassica oleracea var. capitata, Celosia argentea, Coriandrum sativum, Corchorus capsularis, C. olitorius, Ipomoea aquatica, Lactuca scariola var. sativa, Malva verticillata, Raphanus sativus, Spinacea oleracea, Trachyspermum ammi and Trigonella foenum-graceum. Most of these species are cultivated throughout the country except Celosia argentia, Malva verticillata and Trachyspermum ammi. Celosia argentia is cultivated in Rangamati (Kaptai), Sylhet and Patuakhali districts, Malva verticillata is grown in Dinajpur and Rangpur districts, and Trachyspermum ammi is cultivated only in Khagrachari (Golabari) district. Thirty taxa are cultivated for other purposes viz. spice, pulse but leaves of them are also used as vegetables, viz., Allium cepa, A. sativum, Beta vulgaris, Brassica juncea, B. napus, B. rapa, Cajanus cajan, Capsicum frutescens, Cicer arietinum, Colocasia esculenta, Cucurbita maxima, Curcuma longa, Hibiscus cannabinus, Ipomoea batatus, Lablab purpureus, Lageneria siceraria, Lathyrus sativus, Luffa cylindrica, Manihot esculenta, Melocanna baccifiera, Momordica charantia var. muricata, Phaseolus vulgaris, Pisum sativum, Solanum tuberosum, Trachyspermum roxburghianum, Trichosanthes anguina, T. dioica, Vigna mungo, Xanthosoma sagittifolium and Zingiber officinale. In case of tree species usually young leaves are used as vegetables.

Indigenous leafy vegetables can play an important role to alleviate hunger and malnutrition, but they are often neglected in research. They are important sources of micronutrients including Vitamin A and C, iron and other nutrients and are sometimes better nutritional sources than the modern vegetables. Wild leafy vegetables do not warrant any health hazard as they are free from any insecticide, herbicide and pesticides as well as free from the application of chemical fertilizers. Therefore, wild leafy vegetables are superior to the cultivated ones, if they are more or 


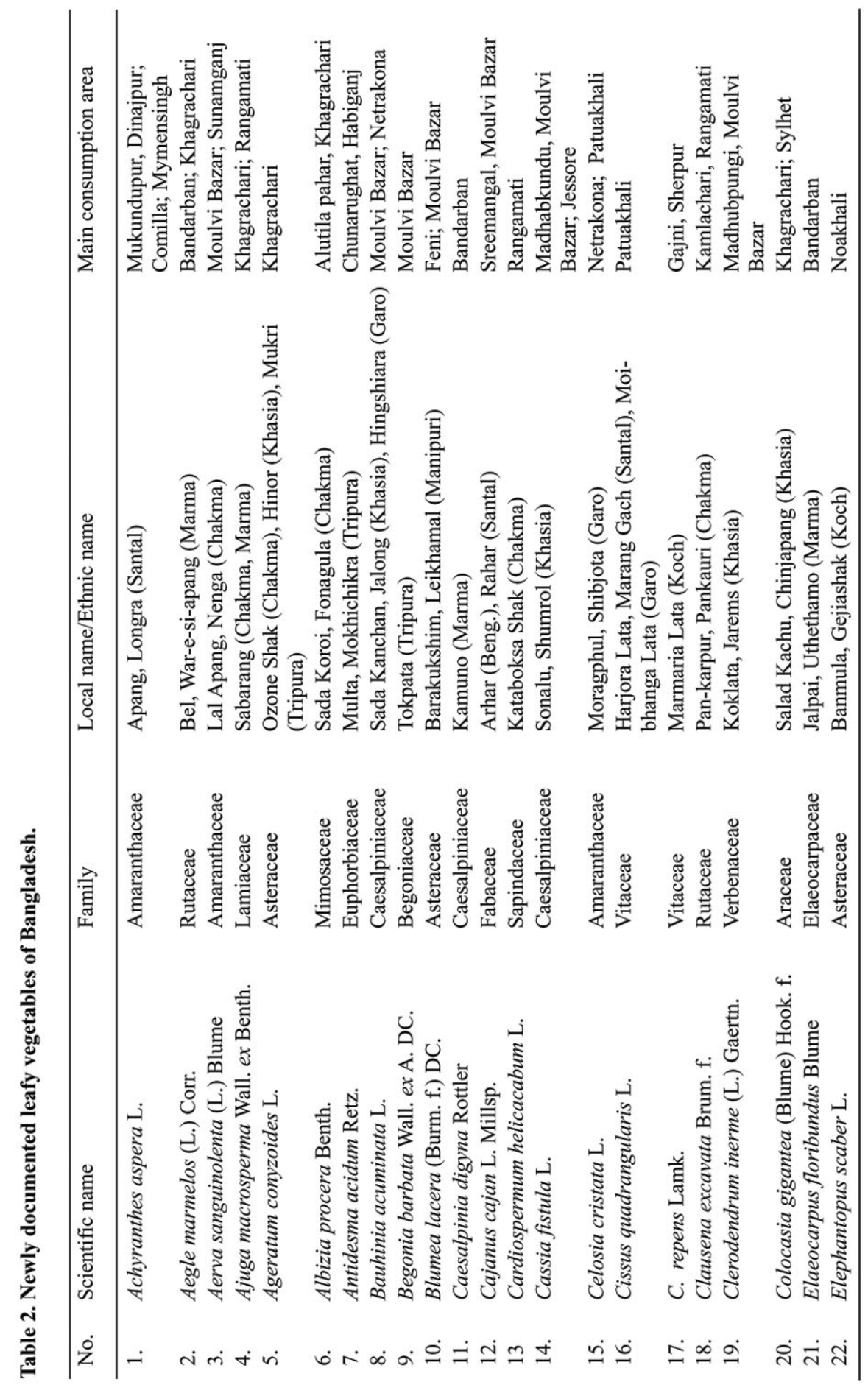




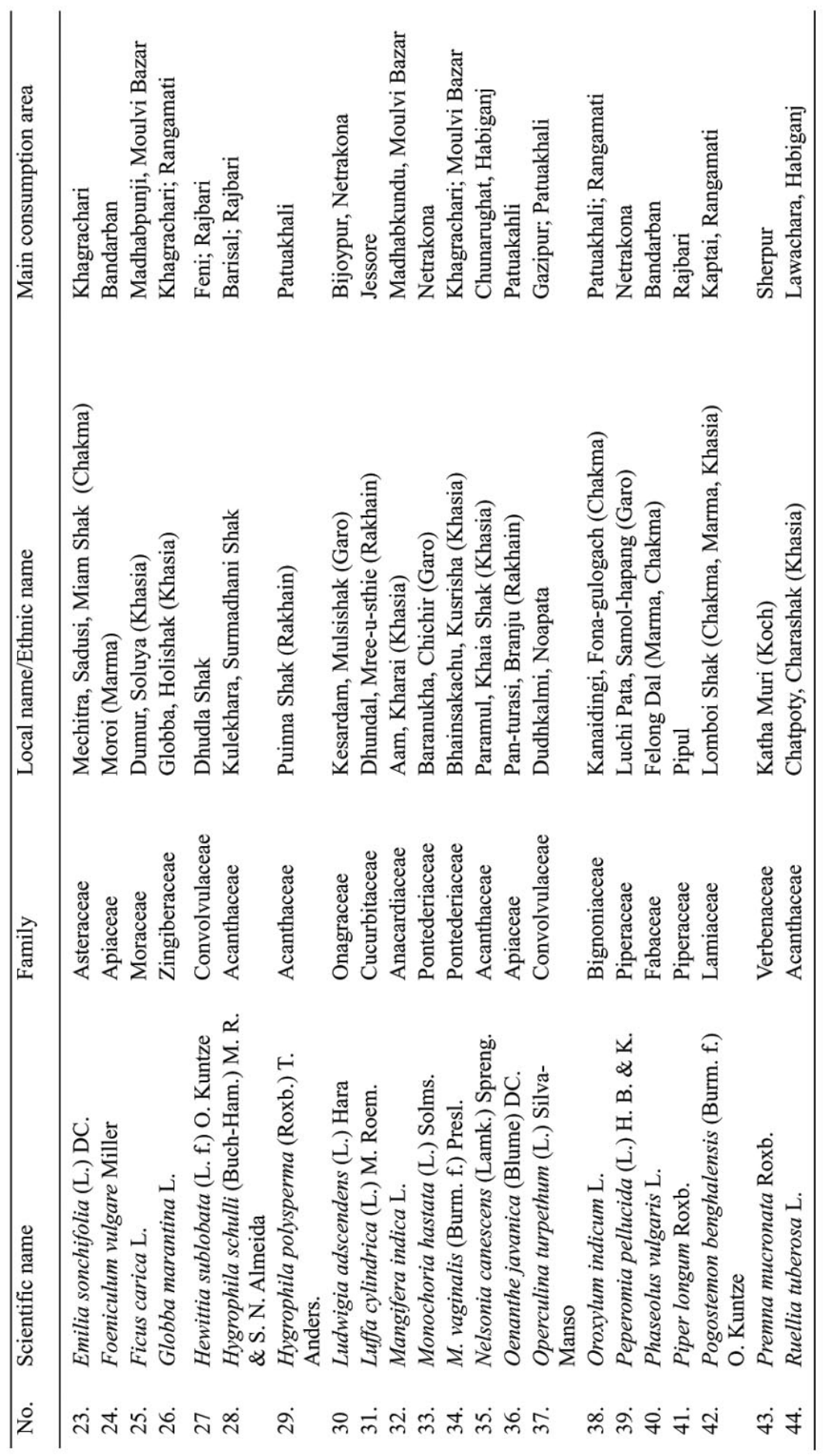




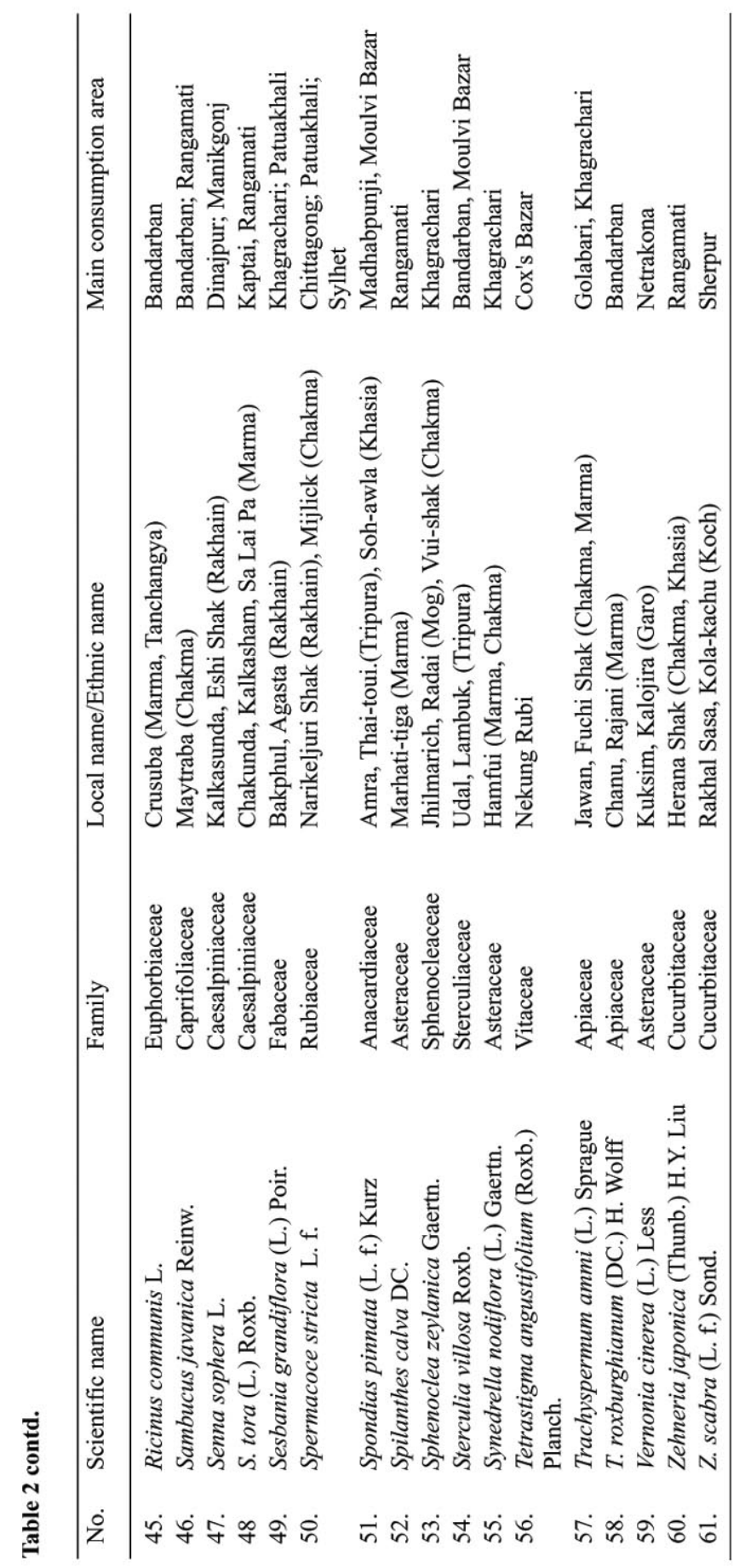


less similar in nutritional values. Despite leafy vegetables have continuously been neglected for long time by the elite people, specially of urban societies, and has been referred to as "Shak, the poor men's food", now with the advancement of scientific research on their nutritive and medicinal values, have become an important item of our daily diet. Edible plants are thought not to contain any risk factors, therefore research for wild edible plants is necessary specially for famine situation and for the people living in rural and forest areas.

The present study on the leafy vegetables of Bangladesh is the most comprehensive study in the country. Extensive botanical exploration throughout the country over ten years resulted in documentation of 61 new reports as leafy vegetables for Bangladesh presenting 30.8\% new addition to the species used as leafy vegetables. This provides a baseline study that could through more light on further research, particularly on nutra-medicinal aspects including determination of proximate nutrients, vitamins, micronutrients, macronutrients. Considring the present study as a baseline, if the nutrient compositions and other nutra-medicinal properties (i.e. antidiabetic, anticancerous, antibacterial and antioxidant) of the leafy vegetables, particularly the newly documented species could be determined, it would be possible to alleviate poverty and malnutrition in Bangladesh through the increased production and consumption of nutritious and health-promoting leafy vegetables.

\section{Acknowledgements}

The authors thank the Director, Bangladesh National Herbarium (DACB) for allowing to work in the herbarium. Thanks are also due to the informants for their help during field visits. A fellowship offered to the first author by the Ministry of Science and Technology is gratefully acknowledged.

\section{References}

Ahmed, Z.U., Hassan, M.A., Begum, Z.N.T., Khondker, M., Kabir, S.M.H., Ahmad, M., Ahmed, A.T.A., Rahman, A.K.A. and Haque, E.U. (Eds). 2008-2009. Encyclopedia of Flora and Fauna of Bangladesh, Vols. 6-10, 12. Asiatic Society of Bangladesh, Dhaka.

Ali, S.M.K., Malek, M.A., Jahan, K. and Salamtullah, Q. (eds). 1977 (reprint 1992). Deshio Khadyo-drobber Pustiman (Nutritional value of local foods). Institute of Nutrition and Food Science, University of Dhaka, Dhaka.

Cronquist, A. 1981. An Integrated System of Classification of Flowering Plants. Columbia University Press, New York, 1262 pp.

Dassanayake, M.D. and Fosberg, F.R. (Eds). 1980-1985. A Revised Handbook to the Flora of Ceylon, Vols.1-6. Amerind Publishing Co. Pvt. Ltd., New Delhi.

Dini, I., Tenore, G.C. and Dini, A. 2005. Nutritional and antinutritional composition of Kancolla seeds: an interesting and underexploited andine food plant. Food Chemistry 92(1): 125-132.

FAO. 2012. The State of Food Insecurity in the World 2012. Rome, Italy.

Fasuyi, A.O. 2006. Nutritional potentials of some tropical vegetable leaf meals: chemical characterization and functional properties. Afr. J. Biotechnol. 5(1): 49-53.

Hassan, M.A. 2010. Deshio Shak Shobjir Pusti Upadhan, Veshojgun o Patthaya Bichar. The Royal Publishers, pp. 1-127.

Hooker, J.D. 1872-1897. The Flora of British India, Vols. 1-7. L. Reeve \& Co. Ltd. Kent, London.

Hyland, B.P.M. 1972. A technique for collecting botanical specimens in rain forests. Flora Malesiana Bulletin 26: 2038-2040.

Kawatra, A., Singh, G. and Sehgal, S. 2001. Nutrition composition of selected green leafy vegetables, hervs and carrots. Plant Foods for Human Nutrition 56: 359-365. 
Khan, M.S. (Ed.) 1972-1987. Flora of Bangladesh. Nos. 1-39. Bangladesh National Herbarium, BARC, Dhaka.

Khan, M.S. and Halim, M. 1987. Aquatic Angiosperms of Bangladesh. Bangladesh National Herbarium, BARC, Dhaka. pp. 1-120.

Khan, M.S. and Rahman, M.M. (Eds). 1989-2002. Flora of Bangladesh, Nos. 40-53. Bangladesh National Herbarium, Dhaka.

Kimura, M. and Rodriguez-Amaya, D.B. 2003. Carotenoid composition of hydroponic leafy vegetables. J. Agric. \& Food Chem. 51: 2603-2607.

Kmiecik, W., Lisiewska, Z. and Jaworska, G. 2001. Effect of storage conditions on the technological value of dill (Anethum graveolens L.). Folia Horticulturea 13: 33-43.

Orech, F.O., Christensen, D.L., Lasen, T., Friis, H., Aagaard-Hansen, J. and Estambale, B.A. 2007. Mineral content of traditional leafy vegetables from western Kenya. Inter. J. Food Sci. \& Nutr. 58(8): 595-602.

Prain, D. 1903 (rep. 1963). Bengal Plants, Vols. 1-2. Botanical Survey of India, Calcutta. pp. 1-1013.

Rashid, M.E. and Rahman, M.A. 2011. Updated nomenclature and taxonomic status of the plants of Bangladesh included in Hook. f., The Flora of British India: Volume-I. Bangladesh J. Plant Taxon. 18(2): 177-197.

Rashid, M.E. and Rahman, M.A. 2012. Updated nomenclature and taxonomic status of the plants of Bangladesh included in Hook. f., The Flora of British India: Volume-II. Bangladesh J. Plant Taxon. 19(2): 173-190.

Reddy, C.V.K. 1999. Greens for Good Health. Nutrition 33(3): 9.

Sarker, S.K. and Hossain, A.B.M.E. 2009. Pteridophytes of greater Mymensingh district of Bangladesh used as vegetables and medicines. Bangladesh J. Plant Taxon. 16(1): 47-56.

Saxena, R. 1999. How green is your diet? Nutrition 33(3): 9.

Siddiqui, K.U., Islam, M.A., Ahmed, Z.U., Begum, Z.N.T., Hassan, M.A., Khondker, M., Rahman, M.M., Kabir, S.M.H., Ahmed, A.T.A., Rahman, A.K.A. and Haque, E.U. (eds). 2007-2008. Encyclopedia of Flora and Fauna of Bangladesh, Vols. 5, 11. Asiatic Society of Bangladesh, Dhaka.

Su, Q., Rowley, K.G., Itsiopoulos, C. and O’Dea, K. 2002. Identification and quantitation of major carotenoids in select components of the Mediterranean diet: Green leafy vegetables, figs and olive oil. European J. Clin. Nutr. 56: 1149-1154.

Sundriyal, M. and Sundriyal, R.C. 2001. Wild edible plants of the Sikkim Himalaya: Nutritive values of selected species. Economic Botany 55: 377-390. 